\title{
Effet de l'addition de glycérol à la ration des vaches laitières sur leur production et sur quelques paramètres de leur métabolisme
}

\author{
B Rémond 1, J Rouel 2, A Ollier 1 \\ 1 INRA, laboratoire de recherche sur la lactation et l'élevage des ruminants, \\ Clermont-Ferrand-Theix, 63122 Saint-Genès-Champanelle; \\ ${ }_{2}^{2}$ INRA, Domaine du Roc, 63210 Orcival, France
}

(Reçu le 26 octobre 1990; accepté le 10 janvier 1991)

\begin{abstract}
Résumé - Dans 3 essais ( 57 vaches Holstein en lactation au total), dont 2 réalisés au début de la lactation, du glycérol (190-610 g) a été ajouté quotidiennement à la ration en substitution à la même quantité d'aliment concentré. Les animaux étaient normalement alimentés avec des régimes à base d'ensilage d'herbe ou de foin et de betteraves. L'apport du glycérol n'a modifié ni la production laitière (quantité de lait produite, teneurs en matières grasses, en protéines et en lactose), ni les quantités d'aliments ingérées (mesure dans 1 essai). II a accru la proportion des acides propionique et butyrique dans le mélange des acides gras volatils du jus de rumen (essais 1 et 3), au détriment de celle de l'acide acétique. Dans le plasma sanguin, il a augmenté la concentration du 3hydroxybutyrate et diminué la glycémie (1 essai). Le glycérol ne semble pas susceptible d'éviter les cétoses chez les vaches laitières quand il est utilisé aux doses employées dans nos essais.
\end{abstract}

glycérol / vache laitière / alimentation / production laitière / cétose

Summary - Effect of glycerol supplementation of the diet of dairy cows on milk production and some digestive and metabolic parameters. In 3 trials (57 Holstein lactating cows in total), 2 of which were carried out at the beginning of lactation, $190-610 \mathrm{~g}$ glycerol were added to the ration daily in substitution for the same quantity of concentrate. Animals were fed according to standards, with diets based on grass silage and hay + fodder beet roots. Glycerol supply had no effect on milk yield and composition and on food intake (measured in 1 trial). It increased the proportion of propionic and butyric acids in the volatile fatty acid mixture of the rumen juice to the detriment of acetic acid. In blood plasma, it increased 3-hydroxybutyrate concentration and decreased glycemia (1 trial). Addition of glycerol to the diet does not appear to be effective in avoiding ketosis in dairy cows when used at the same doses as in our trials.

glycerol / dairy cow / feeding / milk production / ketosis 


\section{INTRODUCTION}

Le glycérol, qui est naturellement présent à l'état libre ou estérifié chez les animaux, est une molécule aux propriétés glucogéniques reconnues ( $c f$ revue de TAO et al, 1983). II serait donc susceptible de présenter de l'intérêt dans l'alimentation des ruminants pendant les périodes où leur néoglucogénèse ne suffit pas à satisfaire leurs besoins en glucose : notamment au début de la lactation chez les vaches fortes productrices, et pendant la fin de la gestation chez les brebis portant plusieurs fœtus.

L'utilisation du glycérol comme additif de la ration des vaches laitières (ajout de $150 \mathrm{~g}$ à $500 \mathrm{~g} / \mathrm{j}$ ) n'a fait l'objet que de 2 essais, au Canada (Fisher et al, 1971; Sauer et al, 1973; Fisher et al, 1973). Dans leurs conclusions, les auteurs mettaient en doute l'efficacité du glycérol utilisé ainsi, alors que son aptitude à relever la glycémie de vaches cétosiques avait été préalablement bien montrée par Johnson (1954) qui en apportait des quantités importantes (900 g à $2250 \mathrm{~g} / \mathrm{j})$ dans la bouche, dans le rumen, ou mélangées aux céréales distribuées, pendant quelques jours. Les études en étaient restées là, car le glycérol était beaucoup plus cher que, par exemple, le monopropylène glycol dont les propriétés néoglucogéniques étaient bien établies (cf revue de Rémond et al, 1984). Cependant, l'utilisation d'huiles végétales comme carburant, après méthanolisation, qui est maintenant au point, entraînerait la mise sur le marché, comme co-produit, de glycérol "technique» en quantité voisine de $10 \%$ de la quantité d'huile traitée, à un prix beaucoup plus bas que celui du glycérol actuellement produit.

Nous avons donc voulu, dans 3 essais, compléter les quelques données à caractère zootechnique acquises sur les effets du glycérol ajouté à la ration des vaches laitières, en utilisant des fourrages à priori susceptibles de favoriser l'apparition de cétoses: de l'ensilage d'herbe dans 2 essais, et des betteraves dans le troisième, car ils favorisent la production, dans le rumen, d'acétate et de butyrate (respectivement) qui sont cétogènes.

\section{MATÉRIEL ET MÉTHODES}

\section{Animaux et schémas expérimentaux}

Seize vaches primipares, en stabulation libre, vêlant en moyenne à 3 ans, ont été utilisées dans l'essai 1 , et 25 vaches multipares, en stabulation entravée, vêlant en moyenne à 5 ans, dans l'essai 2. Elles ont été conduites de la même façon au cours des dernières semaines de la gestation et des 7 premiers j de la lactation. Au $8^{\theta} \mathrm{j}$, elles ont été réparties en 2 lots semblables dont les animaux étaient destinés, pendant les semaines 2 à 7 , pour l'un, à continuer à être normalement alimentés (lot témoin) et pour l'autre à recevoir $240 \mathrm{~g}$ de matière sèche (MS) de glycérol/j en substitution isopondérale à l'aliment concentré.

L'essai 3 a été conduit avec 16 vaches primipares en stabulation libre à partir, en moyenne, de la $9^{\theta}$ semaine de la lactation. Après 2 semaines d'alimentation identique (période préexpérimentale), les animaux ont été répartis en 2 lots semblables destinés à recevoir pendant 4 semaines, en substitution isopondérale (MS) à l'aliment concentré distribué, 200 ou $600 \mathrm{~g}$ de glycérol/j. Après la période expérimentale, tous les animaux ont été alimentés de façon identique pendant 2 semaines. Dans les 3 essais, les vaches étaient de race Holstein.

\section{Alimentation}

Dans les essais 1 et 3 , les vaches (primipares) ont reçu à volonté un ensilage d'un mélange d'herbe de prairie naturelle, de ray-grass anglais et de dactyle, conservé avec 3,9 I d'acide formique + formol et $50 \mathrm{~kg}$ de pulpes sèches de betteraves/t. Sa teneur en MS était de $22 \%$ et celle 
en matières azotées totales (MAT) de $171 \mathrm{~g} / \mathrm{kg}$ de MS. Dans l'essai 2, les vaches (multipares) ont reçu quotidiennement $4 \mathrm{~kg}$ de foin de dactyle de $2^{\mathrm{e}}$ coupe (176 g MAT/kg MS), $4 \mathrm{~kg}$ de MS de betteraves demi-fourragères, ainsi que du foin de prairie naturelle de $1^{r e}$ coupe $(117 \mathrm{~g}$ MAT/kg de MS) à volonté.

La quantité de concentré de production distribuée (186 ou $216 \mathrm{~g}$ MAT/kg MS) a été augmentée au début de la lactation de $1 \mathrm{~kg}$ tous les $3 \mathrm{j}$, de façon à satisfaire les besoins théoriques des animaux, calculés d'après la production laitière de la $1^{\text {re }}$ semaine. Dans l'essai 3 , la quantité de concentré distribuée a été calculée en fonction de la production laitière réelle des vaches. Les teneurs moyennes en MAT des régimes pendant les périodes expérimentales ont été de $20,0,15,2$ et $17,8 \%$ de la MS respectivement pour les essais 1,2 et 3 .

Le glycérol était distribué le matin vers 8 h 30 , en une seule fois, sous forme d'une solution aqueuse dont il constituait $79 \%$ du poids et qui était versée sur le concentré dans les essais 1 et 2 , et sur l'ensilage dans l'essai 3.

\section{Mesures et analyses}

La quantité de lait produite a été mesurée tous les jours et ses teneurs en matières grasses, en protéines, et en lactose, $2 \mathrm{j} / \mathrm{semaine}$ sur un échantillon pondéré des 2 traites. Les quantités de concentré distribuées ont été pesées tous les jours. Les quantités de fourrages ingérées n'ont été mesurées, $4 \mathrm{j} / \mathrm{semaine}$, que dans l'essai 2 où les animaux étaient entravés.

Du jus de rumen a été prélevé, $2-3 \mathrm{~h}$ après la distribution du glycérol, à tous les animaux des essais 1 et 3 , à la fin de la période expérimentale, grâce à une aiguille enfoncée dans le flanc des animaux, en vue du dosage des acides gras volatils (AGV) par chromatographie en phase gazeuse. Du sang a été prélevé dans la queue, $2-4 \mathrm{~h}$ après la distribution du glycérol, à tous les animaux des essais 1 et 2 , en vue du dosage du glycérol (Wieland et Suyter, 1957), du glucose (Merckotest, Postfach 4119, 6100 Darmstadt 1, RFA) et du 3-hydroxybutyrate (Williamson et Mellanby, 1974) dans le plasma.

Les résultats zootechniques ont été soumis à une analyse de variance et de covariance en utilisant, comme covariable, le même critère que celui qui était étudié, mesuré au cours de la période préexpérimentale (essais 1 et 2 ) ou postexpérimentale (essai 3 ). Les résultats sur le sang et sur le jus de rumen ont été soumis à l'analyse de variance à une voie ou au test de Mann-Whitney (Snedecor et Cochran, 1964). Une vache a été retirée de l'essai 1 pour une boiterie très handicapante. Dans l'essai 3,5 vaches dont 4 du même lot ont été atteintes de grippe, et seules les 2 dernières semaines expérimentales ont pu être exploitées. Pour les essais 1 et 2, nous avons considéré comme expérimentaux les résultats des semaines 3 à 7 .

\section{RÉSULTATS ET DISCUSSION}

Aucune vache n'a présenté de signe de cétose. L'apport de glycérol n'a pas provoqué de différences de quantité de lait produite ou de composition du lait entre les 2 lots de chacun des 3 essais (tableau I). II en est de même lorsque les 3 essais sont analysés simultanément $(P>10 \%$ pour tous les critères). L'apport de glycérol ne semble donc pas présenter d'intérêt lorsqu'il est ajouté à raison d'environ 200 ou $600 \mathrm{~g} / \mathrm{j}$ à la ration de vaches normalement alimentées et en bonne santé. Fisher et al (1973) étaient arrivés aux mêmes conclusions pour des apports de glycérol d'environ $180 \mathrm{~g}$ et $360 \mathrm{~g} / \mathrm{j}$. Nous avions également constaté l'absence d'effet du monopropylène glycol (MPG), molécule à laquelle le glycérol a été comparé dans les 2 essais canadiens (Fisher et al, 1971 et 1973), sur la production laitière de troupeaux normalement conduits ( $c$ Rémond et al, 1984). Nous n'avons pas non plus, dans l'essai 2, observé d'effet sur la quantité de fourrage ingérée (tableau I), ce qui est en opposition, au moins apparente, avec l'effet stimulant sur l'appétit observé par Fisher et al (1971). Cependant, ces auteurs avaient enregistré cette stimulation alors que le glycérol était comparé au MPG, qui a tendance à provoquer une diminution de l'ingestion d'aliments que nous 
$\infty \infty$ 으웅유 웅 लं तो in $+1+1+1+1+1+1$ ล ผ $\frac{0}{0} \frac{0}{+1}$

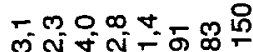
$+1+1+1+1+1+1$ 怘

WRER

ㄴ.

$+1+1+$ ํํำ

$\infty-\infty$

$+1+1+1$

लำ

तल लिख

10. $+1+1+1+1+1+1+1$

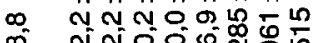

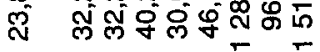

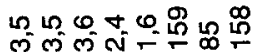
$+1+1+1+1+1+1+1+1$

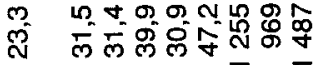

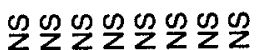

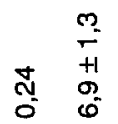

ช $+1+1+1+1+1+1+1+1$

m. w $+1+1+1+1+1+1+$ $N$
$N$
$N$
$N$ 至

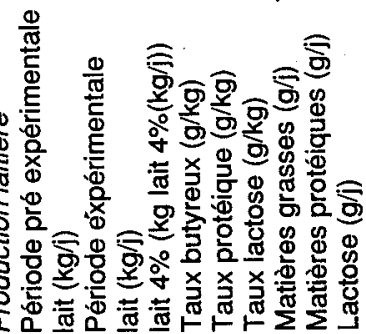


avions déjà remarquée (cf Rémond et al, 1984).

L'apport de glycérol (essai 1) et l'accroissement de sa quantité distribuée (essai 3) dans la ration ont entraîné une augmentation d'une amplitude semblable des proportions des acides propionique et butyrique dans le mélange des AGV du jus de rumen (tableau II), au détriment de celle de l'acide acétique. Calculées par régression linéaire, les modifications des proportions molaires ont été respectivement de $+7,0,+8,1$, et $-15,5$ mol pour 100 par $\mathrm{kg}$ de glycérol ajouté à la ration. Ces résultats sont en accord partiel avec ceux de Fisher et al (1971) qui, chez des vaches recevant un régime constitué de foin et d'aliment concentré, avaient également observé une diminution de la proportion de l'acide acétique et un accroissement de celle de l'acide butyrique mais pas de celle de l'acide propionique. La comparaison est cependant délicate car cet essai comparait les effets de 4 molécules, dont le glycérol, mais ne comportait pas de lot témoin. Nos résultats contrastent toutefois avec ceux obtenus in vitro ou in vivo par d'autres auteurs (Johns, 1953; Garton et al, 1961; Czerkawski et Breckenridge, 1972) qui avaient observé que l'acide propionique était le principal $A G V$ issu de la fermentation du glycérol. Dans ces essais, les niveaux d'alimentation étaient probablement beaucoup plus faibles que dans les nôtres ou dans celui de Fisher et al (1971), puisque les animaux utilisés n'étaient pas en production; par ailleurs, les animaux et la population microbienne de leur rumen n'avaient pas été habitués au glycérol avant les mesures. Peut-être faut-il voir dans l'une ou l'autre de ces différences de protocole la raison des divergences des résultats. Nos résultats concernant la modification du mélange des $A G V$, diffèrent aussi complètement de ceux observés consécutivement à l'apport de MPG. En effet, d'après les résultats de 5 essais enregistrés chez des vaches, des chèvres et des moutons, et récapitulés par Rémond et al (1984), on peut calculer, en rapportant les quantités de MPG distribuées à un poids d'animal de $600 \mathrm{~kg}$, que l'apport de $1 \mathrm{~kg}$ de cette substance entraîne une augmentation systématique de la proportion de propionate de $15,8 \mathrm{~mol}$ pour 100 en moyenne, et une diminution des proportions d'acétate et de butyrate de 12,3 et $4,5 \mathrm{~mol}$ pour 100 respectivement.

Les modifications de la composition du mélange des AGV que nous avons enregistrées expliquent probablement l'accroissement significatif de la teneur du sang en 3-hydroxybutyrate (3-HB) dans les 2 essais où il a été dosé (tableau II), puisqu'il est bien établi qu'une partie de ce composé cétonique provient du butyrate, après sa transformation dans la paroi du rumen au cours de l'absorption. Les chercheurs canadiens avaient également observé un accroissement de ce constituant dans un de leurs essais (Fisher et al, 1971), mais pas dans l'autre (Sauer et al, 1973). Cet accroissement du 3-HB est cohérent avec la forte diminution de la glycémie dans l'essai 2 (où la composition du mélange des AGV n'a malheureusement pas été mesurée) puisque, au début de la lactation au moins, les teneurs du sang en 3-HB et en glucose sont liées de façon négative étroite (Coulon et al, 1985). Dans les essais sur l'utilisation du MPG, cette molécule n'accroissait la glycémie que lorsque celle-ci était faible (cf revue de Rémond et al, 1984) alors que le glycérol semble pouvoir entraîner sa diminution. II faut cependant noter que les auteurs canadiens n'ont pas noté de modifications de la glycémie.

La fréquence de détection du glycérol dans le plasma sanguin n'a pas été différente entre les 2 lots dans les essais 1 et 2 où il a été dosé. Cela n'est pas surprenant puisqu'au moins une partie du glycérol in- 


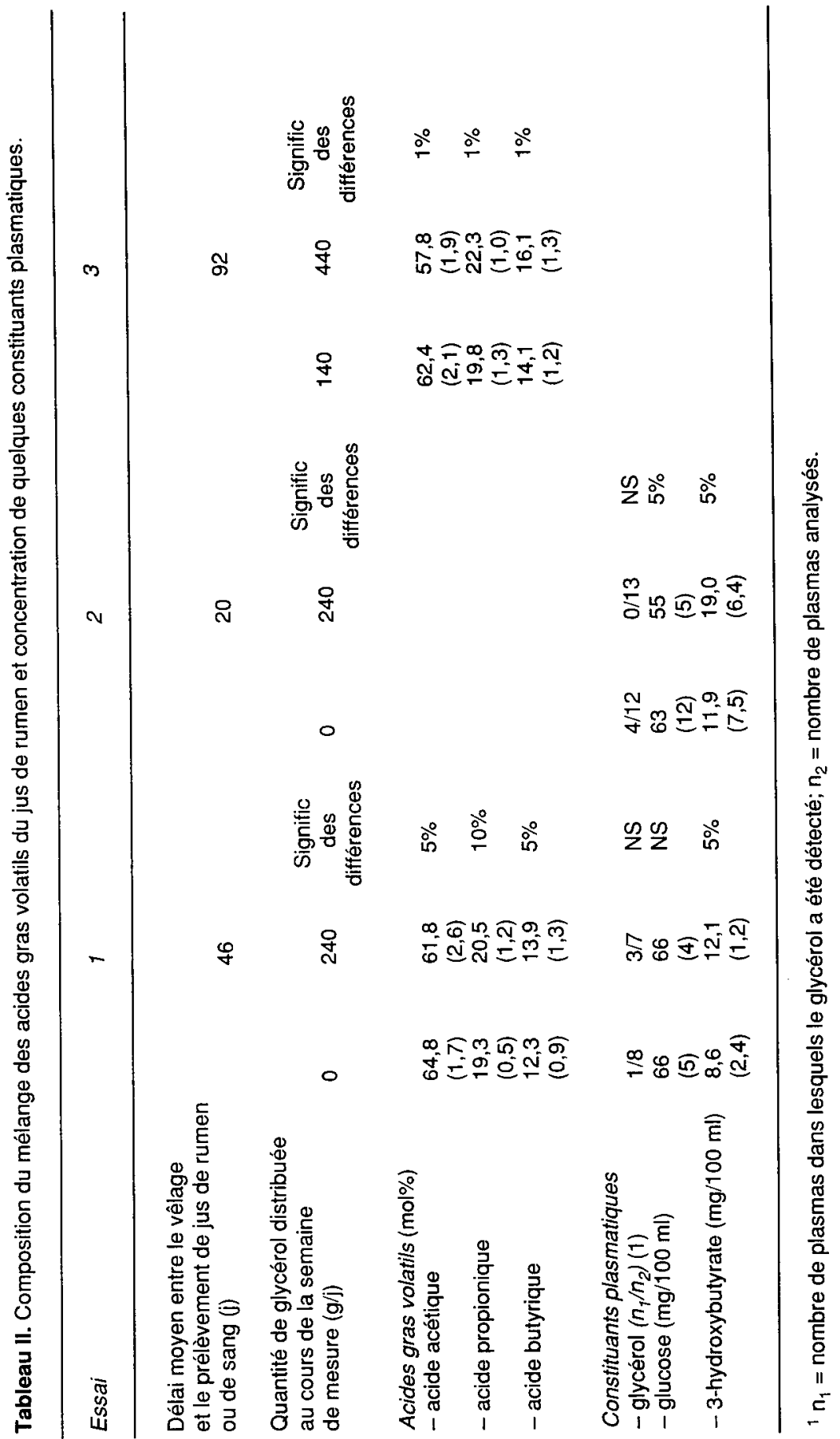


géré est fermenté dans le rumen (cf cidessus), le foie capte la presque totalité du glycérol circulant (cf revue de Tao et al, 1983), et le temps de renouvellement du pool de glycérol libre est faible (Bergman, 1968).

\section{CONCLUSION}

L'addition quotidienne de $200-600 \mathrm{~g}$ de glycérol à des rations équilibrées pour vaches laitières ne semble pas présenter d'intérêt. Pour apprécier l'aptitude du glycérol à limiter l'incidence des cétoses, il aurait fallu expérimenter avec des animaux dont une fraction, la plus élevée possible, aurait présenté cette maladie, ce qui n'a été le cas d'aucune vache des 3 essais. Cependant, les modifications digestives et métaboliques qu'il entraîne lorsqu'il est ajouté quotidiennement et en faible quantité à la ration, lui ôtent toute probabilité d'être un additif protecteur contre les cétoses. Cela contraste avec ce que laissaient supposer :

- son effet curatif clairement établi lorsqu'il est administré à des doses élevées $(0,9-$ $2,3 \mathrm{~kg} / \mathrm{j}$ ) et pendant des périodes de quelques jours à des vaches cétosiques (Johnson, 1954);

- la production préférentielle de propionate à laquelle donne lieu sa fermentation dans le rumen, selon la majorité des résultats acquis antérieurement (voir ci-avant).

\section{REMERCIEMENTS}

Nous remercions l'Organisation nationale interprofessionnelle des oléagineux (Onidol), 12, avenue Georges V, 75008 Paris, qui a participé au financement de cette étude, Monsieur A François, directeur de recherches honoraire à I'INRA qui l'a suscitée et encouragée, C Celaudoux et les techniciens animaliers du domaine
INRA d'Orcival pour leurs soins aux animaux, $J$ Fléchet et $R$ Lefaivre pour les analyses chimiques, Y Fournier pour la dactylographie.

\section{RÉFÉRENCES}

Bergman EN (1968) Glycerol turnover in the non-pregnant and ketotic pregnant sheep. Am J Physiol 215, 865-873

Coulon JB, Rémond B, Doreau M, Journet $M$ (1985) Évolution de différents paramètres sanguins du métabolisme énergétique chez la vache laitière en début de lactation. Ann Rech Vét 16, 185-193

Czerkawski JW, Breckenridge G (1972) Fermentation of various glycolytic intermediates and other compounds by rumen micro-organisms, with particular reference to methane production. Br J Nutr 27, 131-146

Fisher LJ, Erfle JD, Sauer FD (1971) Preliminary evaluation of the addition of glycogenic materials to the rations of lactating cows. Can $J$ Anim Sci 51, 521-727

Fisher LJ, Erfle JD, Lodge GA, Sauer FD (1973) Effects of propylene glycol or glycerol supplementation of the diet of dairy cows on feed intake, milk yield and composition, and incidence of ketosis. Can J Anim Sci 53, 289296

Garton GA, Lough AK, Vioque E (1961) Glyceride hydrolysis and glycerol fermentation by sheep rumen contents. J Gen Microbiol 25, 215-225

Johns AT (1953) Fermentation of glycerol in the rumen of sheep. NZJ Sci Tech 35, 262-269

Johnson RB (1954) The treatment of ketosis with glycerol and propylene glycol. Cornell Vet 44, 6-21

Rémond $B$, Rémésy $C$, Ruffio $P$, Chilliard $Y$ (1984) Intérêt du monopropylène glycol dans la prévention et dans le traitement des cétoses chez les vaches laitières. Bull Tech CRZV Theix, INRA 56, 21-30

Sauer FD, Erfle JD, Fisher LJ (1973) Propyleneglycol and glycerol as a feed additive for lactating dairy cows: an evaluation of blood metabolite parameters. Can J Anim Sci 53, 265271 
Snedecor GW, Cochran WG (1964) Statistical Methods. 6th edn. The lowa State Univ Press, Ames, lowa, États-Unis

Tao RC, Kelly RE, Yoshimura NN, Benjamin F (1983) Glycerol: its metabolism and use as an intravenous energy source. I Parenter Enterol Nutr 7, 479-488
Wieland O, Suyter M (1957) Glycerokinase: Isolierung und Eigenschaften des Enzyms. Biochem Z 329, 320-331

Williamson DH, Mellanby J (1974) D(-) Bhydroxybutyrate. In: Methods of Enzymatic Analysis (HU Bergmeyer, ed), $2^{\theta}$ edn, vol 4, 1836-1839, Acad Press, New York 